ARTICLE

Received 11 Sep 2014 | Accepted 18 Feb 2015 | Published 26 Mar $2015 \quad$ DOl: 10.1038/ncomms7679

\title{
Fundamental origins and limits for scaling a maternal morphogen gradient
}

Feng $\mathrm{He}^{1, \star}$, Chuanxian Wei ${ }^{1,2,3, \star}$, Honggang Wu ${ }^{1,2,3}$, David Cheung ${ }^{1}$, Renjie Jiao ${ }^{2,4} \&$ Jun Ma $a^{1,5}$

Tissue expansion and patterning are integral to development; however, it is unknown quantitatively how a mother accumulates molecular resources to invest in the future of instructing robust embryonic patterning. Here we develop a model, Tissue Expansion-Modulated Maternal Morphogen Scaling ( $\mathrm{TEM}^{3} \mathrm{~S}$ ), to study scaled anterior-posterior patterning in Drosophila embryos. Using both ovaries and embryos, we measure a core quantity of the model, the scaling power of the Bicoid (Bcd) morphogen gradient's amplitude $n_{A}$. We also evaluate directly model-derived predictions about Bcd gradient and patterning properties. Our results show that scaling of the Bcd gradient in the embryo originates from, and is constrained fundamentally by, a dynamic relationship between maternal tissue expansion and bcd gene copy number expansion in the ovary. This delicate connection between the two transitioning stages of a life cycle, stemming from a finite value of $n_{A} \sim 3$, underscores a key feature of developmental systems depicted by $\mathrm{TEM}^{3} \mathrm{~S}$.

\footnotetext{
${ }^{1}$ Division of Biomedical Informatics, Cincinnati Children's Research Foundation, 3333 Burnet Avenue, Cincinnati, Ohio 45229, USA. ${ }^{2}$ State Key Laboratory of Brain and Cognitive Science, Institute of Biophysics, Chinese Academy of Sciences, 15 Datun Road, Beijing 100101, China. ${ }^{3}$ University of Chinese Academy of Sciences, Beijing 100080, China. ${ }^{4}$ Sino-French Hoffmann Institute, Guangzhou Medical University, 195 Dongfengxi Road, Guangzhou 510182, China. ${ }^{5}$ Division of Developmental Biology, Cincinnati Children's Research Foundation, 3333 Burnet Avenue, Cincinnati, Ohio 45229, USA. * These authors contributed equally to this work. Correspondence and requests for materials should be addressed to J.M. (email: jun.ma@cchmc.org).
} 
S caling of biological activities with organismal size is a general property of life ${ }^{1}$; however, it is actively debated whether all life forms and biological activities follow a single universal scaling relationship ${ }^{2-7}$. From the perspective of developmental biology, scaling is about proportionality of tissue/organ size to the overall body size, which is one of the most intriguing but poorly understood features of animal development ${ }^{8-10}$. Two aspects of the developmental scaling problem, formation of scaled patterns and expansion of specified tissues, have been investigated intensively ${ }^{11-20}$. To provide a unified perspective suitable for evaluating diverse systems at different levels, we can define the relationship between tissue specification (patterning) and tissue expansion (growth) of a given developmental system based on how they are connected temporally within a time period of interest. These two events can take place either concurrently or with one preceding the other, representing three basic types of temporal relationships or temporal logics (Fig. 1a). In logic a, such as the Drosophila wing disc, tissue patterning and expansion are concurrent events that could also (but do not have to) be coupled mechanistically ${ }^{21,22}$. In logic $\mathbf{b}$, such as the increase in an animal's muscle mass, patterning of a tissue precedes its expansion ${ }^{13,20}$. In logic c, such as the Drosophila embryo, patterning takes place when size has been pre-determined ${ }^{23}$.

Our current work concerns temporal logic c. In Drosophila, patterning along the anterior-posterior (AP) axis of the embryo is instructed by the maternal morphogen gradient Bicoid $(\mathrm{Bcd})^{24-28}$. A well-recognized feature of this system is the robust and scaled patterning outcome ${ }^{11,12}$; however, its underlying mechanisms remain a topic of active investigation. Our recent studies have led to the documentation that the Bcd morphogen gradient in Drosophila melanogaster embryos possesses scaling properties ${ }^{14,23,29}$. We found that a general property of the embryo relevant to Bcd gradient scaling is that the amount of maternally deposited $b c d$ mRNA is correlated with embryo size $e^{23,29}$. However, the precise origin of such a correlation remains unknown. In addition, although one of our documented within-species scaling mechanisms has a resemblance to a between-species scaling mechanism ${ }^{29,30}$, it represents a special case involving abnormal bcd mRNA localization in the embryo. Thus, we are currently lacking a unified mechanistic view of the maternal origins and evolutionary conservation of $\mathrm{Bcd}$ gradient scaling in the embryo.

In this report we establish, and experimentally advance, a framework designed to evaluate the origins and limits of Bcd gradient scaling within a species. Our framework is referred to as the Tissue Expansion-Modulated Maternal Morphogen Scaling $\left(\mathrm{TEM}^{3} \mathrm{~S}\right)$ model. This model unifies specifically and quantitatively the properties and events of maternal tissue expansion and scaled embryonic patterning under temporal logic c. We perform independent measurements to estimate a core quantity of this model, the scaling power of Bcd gradient's amplitude $n_{A}$. We also perform measurements to directly evaluate model-derived predictions in the embryo. We show that, in addition to connecting the events that take place in two distinct stages of Drosophila life cycle, the $\mathrm{TEM}^{3} \mathrm{~S}$ model also provides a unified view of the two distinct scenarios of Bcd gradient scaling (that is, within-species versus across-species) from an evolutionary perspective at a mechanistic level.

\section{Results}

The TEM ${ }^{3} S$ model. We establish a general framework of biological scaling in a developmental system that follows temporal logic c (Fig. 1a). Here tissue expansion takes place in a biological entity referred to as system $\mathbf{E}$, while patterning takes place in a distinct (but connected by the life cycle) entity referred to as system P. We base our model on the Drosophila morphogen gradient of Bcd. Thus, tissue expansion in our model refers specifically to the growth of an egg chamber in the ovary of the mother (system E) and scaled spatial patterning is a property that is specific to the future embryo (system $\mathbf{P}$ ). One of our objectives is to build a unified conceptual framework within which we can compare model-derived predictions with observed properties of the actual biological systems. In our theoretical discussions that are further detailed in Supplementary Notes 1-4, we may on occasions choose to use parameter values that are idealistic, but they are consistent with the actual biological system in hand. Importantly, our general conclusions about the expected behaviour of developmental systems depicted by the $\mathrm{TEM}^{3} \mathrm{~S}$ model do not depend on particular parameter values chosen for analysis.

In previous studies of the Bcd morphogen gradient, a patterning system's length $L$ is usually treated as a given value when the gradient behaviours are evaluated ${ }^{31-38}$. In our discussion, we treat $L$ as a property that is inherited specifically from system E. Under temporal logic c, $L$ is predetermined by system $\mathbf{E}$, representing a variable that is independent of space or time in system $\mathbf{P}$. This feature makes the Bcd gradient different from morphogen systems that follow other temporal logics. Since our discussion focuses on scaling, we express a given position $x$ along the length of system $\mathbf{P}$ as a relative value $\xi=x / L$.

The $\mathrm{TEM}^{3} \mathrm{~S}$ model formalizes the scaling feature of patterning by stating that a morphogen gradient possesses properties necessary for it to achieve a performance objective in system $\mathbf{P}$. Performance objective is defined as attaining one (or more) relative position $\xi$, at which morphogen concentration $M$ is insensitive to fluctuations in $L$. This necessitates a search for a solution(s) of $\xi$ to the first-order partial differentiation with respect to $L$ being zero as

$$
\frac{\partial M(L, \xi, t)}{\partial L}=0 .
$$

We denote the solution $\xi=\xi_{\mathrm{C}}$ as the critical position of system $\mathbf{P}$. This solution could be a function of $t$ (that is, scaling is timedependent); however, by definition it is independent of $L$. To be a realistic value, $\xi_{C}$ must be within the physical boundary [0 1 l] of system $\mathbf{P}$.

The Bcd gradient profile may be approximated by an exponential function ${ }^{11,26}$. Here we consider a two-parameter model of an exponential morphogen gradient that is stable over time, $M(\xi, L)=A e^{-\Gamma \xi}$. In an idealized $1 \mathrm{D}$ model based on synthesis, diffusion and decay, this gradient represents the steadystate morphogen concentration, where $A=J /(D \omega)^{1 / 2}$ and $\Gamma=L /$ $(D / \omega)^{1 / 2}$, and $J$ is the morphogen production rate from a point source at $\xi=0, D$ is the diffusion constant and $\omega$ is the first-order rate constant of decay (see Supplementary Figs 1-4 and Supplementary Notes 1-4 for additional considerations). In this two-parameter model, either the amplitude $A$ or the slope measurement $\Gamma$ (or both) could be a quantity related to $L$. For our study, we define scaling power, $n_{Q}=\frac{\mathrm{d} Q / Q}{\mathrm{~d} L / L}$, as the normalized derivative of a biological quantity $Q$ with respect to that of the system length $L$. If $n_{\mathrm{Q}}$ can be approximated to a finite constant value with respect to $L$, we have a power-law relationship $Q \propto L^{n_{Q}}$. If $\Gamma$ is independent of $L$, that is, $n_{\Gamma}=0$, it is possible for all positions to satisfy equation (1). If $n_{\Gamma} \neq 0$, there is only one solution to equation (1), $\xi_{\mathrm{C}}=n_{A} / \Gamma n_{\Gamma}$. By definition, this solution determines the critical position, at which $M$ is insensitive to fluctuations in $L$. Under the condition that diffusivity and decay 
a

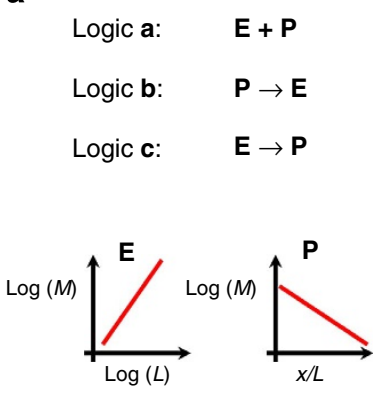

C

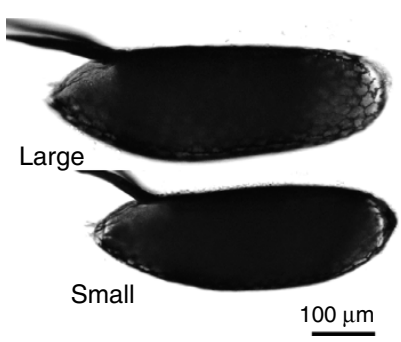

b

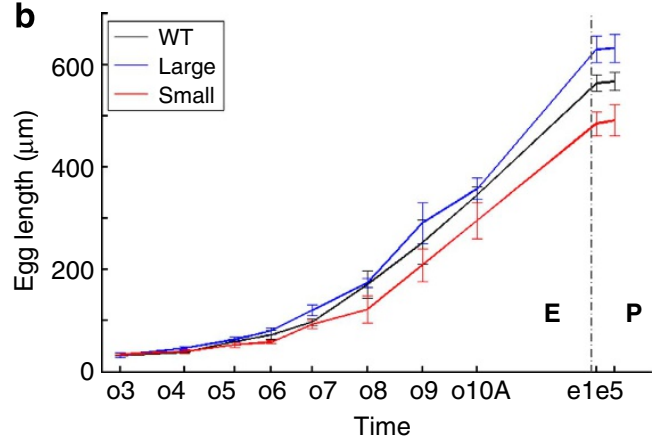

d

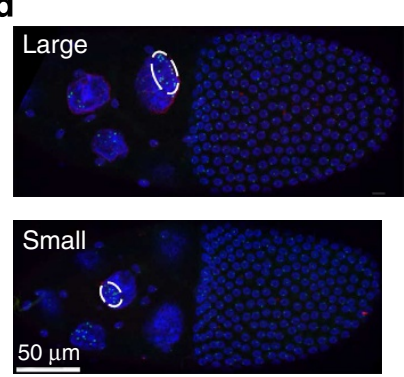

e

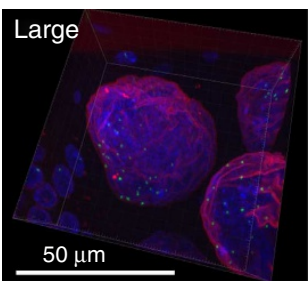

Figure 1 | Investigating tissue expansion properties during oogenesis in Drosophila. (a) Diagrams depicting three basic temporal logics governing the relationships between tissue expansion $(\mathbf{E})$ and patterning $(\mathbf{P})$ within a time period of interest. A symbolic plot capturing each system's fundamental feature is shown. For the depicted system $\mathbf{E}$, where $M$ refers to a given type of molecules (for example, mRNA or protein) that accumulate in quantity in relation to the 1D size, $L$, of the system, the slope shown is the scaling power, $n$, of the molecules. For the depicted system $\mathbf{P}$, where $M$ refers to morphogen molecules that form an exponential concentration gradient along the normalized length, $x / L$, of the patterning system, the slope shown is $-\Gamma$, negative of the system attribute (see Results for additional details). (b) Measured lengths of egg chambers and embryos. WT, Large and Small denote $w^{1118}$, large- and small-egg inbred lines, respectively (shown in black, blue and red, respectively). Error bars are s.d.'s and sample numbers are 50 (embryos) or four or more (egg chambers) for each of the three lines at each of the stages shown. Student's $t$-tests suggest significant between-strain differences in lengths $(P$ values $<0.05)$ as early as oogenesis stage 6. (c,d) Freshly laid eggs $(\mathbf{c})$ or stage-10A egg chambers $(\mathbf{d})$ from inbred lines. White circle in $\mathbf{d}$ marks the cluster of the detected bcd DNA FISH dots (green) within a nurse cell nucleus (DAPI in blue; WGA in red; see Methods). (e) Higher magnification of a stage-10A egg chamber. Scale bars, $100 \mu \mathrm{m}$ (for $\mathbf{c}$ ) and $50 \mu \mathrm{m}$ (for $\mathbf{d , e}$ ).

of morphogen molecules are properties intrinsic to a given species and thus insensitive to $L$, that is, $n_{\Gamma}=1$, we have

$$
\xi_{\mathrm{C}}=\frac{n_{A}}{\Gamma} \text {. }
$$

Equation (2) shows that, for a stable exponential gradient whose slope is independent of $L$, the performance objective of attaining $\xi_{\mathrm{C}}$ could be achieved only if $\Gamma$ is properly matched by the scaling power of a morphogen gradient's amplitude $n_{A}$ (Supplementary Fig. 1 and Supplementary Note 1). For purposes of perspective and convenience, we refer to $\Gamma$, which quantifies the length of system $\mathbf{P}$ in a relative term, as the system attribute. In biochemical terms, $\Gamma$ could be viewed as a constant that exists for a given species and this constant can be evaluated through experimental measurements in the embryos. Equation (2) provides a general expression of the $\mathrm{TEM}^{3} \mathrm{~S}$ model. It states straightforwardly that the behaviour of system $\mathbf{P}$ with regard to the existence and location of its critical position (defined as $\xi_{\mathrm{C}}$ ) is determined by the fundamental properties that connect system $\mathbf{E}$ to system $\mathbf{P}$ (as encapsulated by $n_{A}$-see below) and the length of system $\mathbf{P}$ in relation to the gradient's length scale (as signified by $\Gamma$ ).

A dynamic framework connecting system $\mathbf{E}$ to system $\mathbf{P}$. In the $\mathrm{TEM}^{3} S$ model, both the $1 \mathrm{D}$ size $L$ and the scaling power of the morphogen gradient's amplitude $n_{A}$ in system $\mathbf{P}$ can be viewed as inherited properties in the sense that they are derived from system $\mathbf{E}$ that has ceased to exist but has been physically transitioned into system $\mathbf{P}$. To formally link systems $\mathbf{E}$ and $\mathbf{P}$, we develop a quantitative framework that describes the dynamic relationship between size and scaling power (Supplementary Note 2). In this framework, the morphogen protein in the embryo (system $\mathbf{P}$ ) is the end product in a chain of linear-forward transitions between molecular species that originate from the egg chamber in the ovary (system $\mathbf{E}$ ): gene $\rightarrow$ mRNA $\rightarrow$ protein. Here morphogen gene copies and morphogen protein molecules are unique to systems $\mathbf{E}$ and $\mathbf{P}$, respectively, and morphogen mRNA is the only species that can exist in both systems. As an egg chamber expands its size during oogenesis, morphogen gene copies undergo endoreplication (in nurse cells) and are used as templates for mRNA production (see experimental data below). Using first-order rate constants to describe dynamic tissue growth and chemical reactions, we can deduce that (see Supplementary Note 2),

$$
n_{A} \sim n_{3} \sim n_{2} \sim n_{1} \approx \frac{j_{1}}{k_{1}}
$$

where $n_{1}, n_{2}$ and $n_{3}$ are the scaling powers of each of the molecular species (gene, mRNA and protein, respectively) in the chain with respect to the length of a corresponding biological entity, and $j_{1}$ and $k_{1}$ are first-order rate constants for the expansions of, respectively, morphogen gene copy number and 1D size of a maternal entity (for example, nurse cell nucleus) during oogenesis.

Equation (3) postulates a dynamic origin of the scaling power of the morphogen gradient's amplitude $n_{A}$ in system $\mathbf{P}$. In other words, $n_{A}$, a quantity that is for system $\mathbf{P}$ and core to the $\mathrm{TEM}^{3} \mathrm{~S}$ model, is defined fundamentally by the dynamic properties of system E. Thus, an experimental measurement of the quantity $n_{A}$ 
is important not only to advancing mechanisms of developmental scaling but also to understanding quantitative biological principles that govern the 'accumulation' and 'consumption' of molecular resources in connecting two stages of a life cycle. Equation (3) also states that the scaling power $n_{A}$ can be estimated through the use of independent methods that quantify each of the three molecular species in the linear chain, a postulate to be evaluated directly by experiments.

The experimental system. The Drosophila egg is derived from the maternal tissue ovary (see Methods for definition of oogenesis stages). To advance our $\mathrm{TEM}^{3} \mathrm{~S}$ model experimentally, we first quantified growth properties of the egg chamber in the ovary. In addition to $w^{1118}$ as the wild-type (WT), we used two inbred lines that had been selected to lay large or small eggs ${ }^{23,39}$. Our measurements in the ovary document that egg size divergence between these two lines originates from oogenesis (Fig. 1b-d). Towards quantifying scaling powers during oogenesis, we established whole-mount procedures for the ovary (see Methods and Supplementary Table 1). We used DNA fluorescent in situ hybridization (FISH) to quantify individual gene loci (such as $b c d$ ), DAPI staining to quantify bulk nuclear DNA and wheat germ agglutinin (WGA) staining to visualize the nuclear envelope (Fig. 1d,e). Our whole-mount tissues were processed to maximally preserve their native spatial features. In addition, our confocal imaging was performed within a documented linear range (Supplementary Fig. 5).

A growing egg chamber consists of one oocyte, 15 nurse cells and thousands of follicle cells ${ }^{40-43}$. Nurse cells and follicle cells provide external signals, nutrients and other materials to the oocyte that will become the future egg. Our primary interest of the current work is in nurse cells because it is these cells that produce bcd mRNA; however, for calibration purposes, we used follicle cells. These cells undergo three rounds of endoreplication $^{44,45}$, leading to four well-resolved subpopulations with expected genome polyploidy of $2 \mathrm{C}, 4 \mathrm{C}, 8 \mathrm{C}$ and $16 \mathrm{C}$ (Fig. 2a,b; see Methods). Such calibrations within a given experiment (Fig. 2c,d) permitted us to estimate a nurse cell's genome polyploidy or $b c d$ gene copy number (see Fig. 2e,f for scatter plots of data from individual nurse cell nuclei).

Metric expansion of nurse cell nuclei. Nurse cells undergo three distinct phases of endoreplication ${ }^{46}$ : (1) complete replication during the first five rounds to form a single polytene chromosome, (2) dispersion of the 64 chromosomes into 32 chromatid pairs and (3) additional rounds of endoreplication for each of these 32 polytene chromosomes. The bcd gene loci detected in our whole-mount FISH remain spatially clustered inside the nurse cell nucleus (Fig. 1d,e). Such clusters could reflect the working of 'continued forces' that drive, for example, homologous pairing or interactions with the nuclear architecture to restrict free expansion ${ }^{47}$. Alternatively, the clusters themselves could expand in a manner that is analogous to metric expansion, in which case the existence of such clusters would reflect the working of the 'initial forces' that restricted the dispersion step. Our results obtained from both WT (Fig. 3a) and the inbred lines (Fig. 3b) are consistent with the latter hypothesis and support a volumetric expansion after dispersion.

To investigate the spatial relationship between clusters of different gene loci on a chromosome, we analysed double-FISH data for $b c d$ paired with either nanos (nos) or a chorion protein gene locus (Cp, see Methods) in WT embryos. The detected clusters for nos or $C p$ loci expand similarly to those of $b c d$ inside nurse cell nuclei, supportive of volumetric expansion (Fig. 3a). However, between-cluster expansion ( $b c d$-nos or $b c d-C p)$ exhibits properties suggesting that they are subject to constraints sensitive to the intervening DNA length (Fig. 3a, black squares and grey diamonds; see Methods for additional details and see Supplementary Fig. 6 for spatial properties of gene loci in follicle cell nuclei).

Scaling powers for nuclear DNA and bcd gene copy number. Since the $b c d$ gene locus is a part of the entire genome undergoing endoreplication, we first quantified the bulk nuclear DNA in relation to the expansion of the nurse cell nuclear diameter $l$. We estimated the scaling power for the bulk nuclear DNA $n_{0}$ using the fitted slope in a log-log plot (Fig. 4a). We obtained $n_{0}=2.42$, 2.27 and 2.50 for WT, large- and small-egg lines, respectively (95\% confidence intervals are: $2.32 \sim 2.51,2.04 \sim 2.50$ and $2.22 \sim 2.77$ ). Using all data pooled, $n_{0}=2.43$ (95\% confidence interval $(\mathrm{CI})=2.20 \sim 2.66)$.

To estimate $n_{A}$ using an independent method that is specific to the $b c d$ gene locus, we determined the relationship between $b c d$ gene copy number and nurse cell nuclear diameter $l$. We obtained the scaling power of the $b c d$ gene copy number $n_{1}=2.82,3.10$ and $2.93,2.95$ for WT, large-egg line, small-egg line and three lines pooled, respectively (Fig. $4 \mathrm{~b} ; 95 \% \mathrm{CI}=2.62 \sim 3.03$, $2.78 \sim 3.412 .48 \sim 3.39$ and $2.72 \sim 3.18$ ). These results document that $n_{1}$ is a maternal property that is insensitive to size of the future egg ( $n_{1}$ of the large-egg line is within $95 \% \mathrm{CI}$ of that of the small-egg line and vice versa). They support the deduced relationship in equation (3) where $n_{A}$ is defined fundamentally by rate constants of the maternal processes of $b c d$ gene copy number expansion and tissue expansion.

To determine whether $n_{1}$ obtained for the $b c d$ gene locus is reflective of a general property of an expanding egg chamber in the ovary as postulated (Supplementary Note 2), we measured the scaling power for the locus of nos, another nurse cell-expressing gene. We obtained $n_{1}=2.83,2.79,3.14$ and 2.88 in WT, large-egg line, small-egg line and three lines pooled, respectively (Fig. $4 \mathrm{c}$, $95 \% \mathrm{CI}=2.61 \sim 3.06,2.35 \sim 3.23,2.71 \sim 3.57$ and $2.65 \sim 3.11$ ), values consistent with those measured on $b c d$.

bcd gene copy number at the peak time during oogenesis. At stage 10 of oogenesis, nurse cell nuclei reach their peak size ${ }^{40}$. We estimate that a WT stage-10A egg chamber contains a total of $6.3 \pm 1.2 \times 10^{3}$ copies of the bcd gene in 15 nurse cells combined (Fig. 2f; see Fig. 2e legend for estimated genome polyploidy number at the same stage). We note that this peak $b c d$ gene copy number is very similar to the total number of nuclei of a cellularizing blastoderm embryo. A large number of $b c d$ gene templates in an egg chamber would ensure a reliable production of $b c d$ mRNA to be deposited to the egg as seen in the embryo $^{23,29,48}$. For the large- and small-egg inbred lines, this number is $6.8 \pm 1.4 \times 10^{3}$ and $5.6 \pm 1.1 \times 10^{3}$, respectively. These results document a direct divergence in $b c d$ gene template number in nurse cells of the inbred lines despite the fact that they had been selected based solely on the size of the eggs laid ${ }^{39}$.

Measurements of $\boldsymbol{n}_{\mathbf{2}}$ and $\boldsymbol{n}_{\mathbf{3}}$ in embryos. To further evaluate equation (3), we measured $n_{2}$ and $n_{3}$, the scaling power for $b c d$ mRNA and Bcd protein, respectively, in embryos from the inbred lines that offered an enhanced size spread for effective analysis (Fig. 1b). We obtained $n_{2}=2.70 \quad\left(R^{2}=0.86,95 \% \quad \mathrm{CI}=2.36\right.$ $\sim 3.04)$ and $n_{3}=3.03\left(R^{2}=0.71,95 \% \quad \mathrm{CI}=2.00 \sim 4.06\right.$; see Supplementary Table 2 for idealized and measured parameters of a full morphogen gradient model). Fig. 5a shows an overlay of measurements for all three molecular species within the linear chain. Supportive of equation (3), datapoints from these independent measurements congregate towards a consensus slope (Fig. 5a). Since endoreplication is a regulated process during 

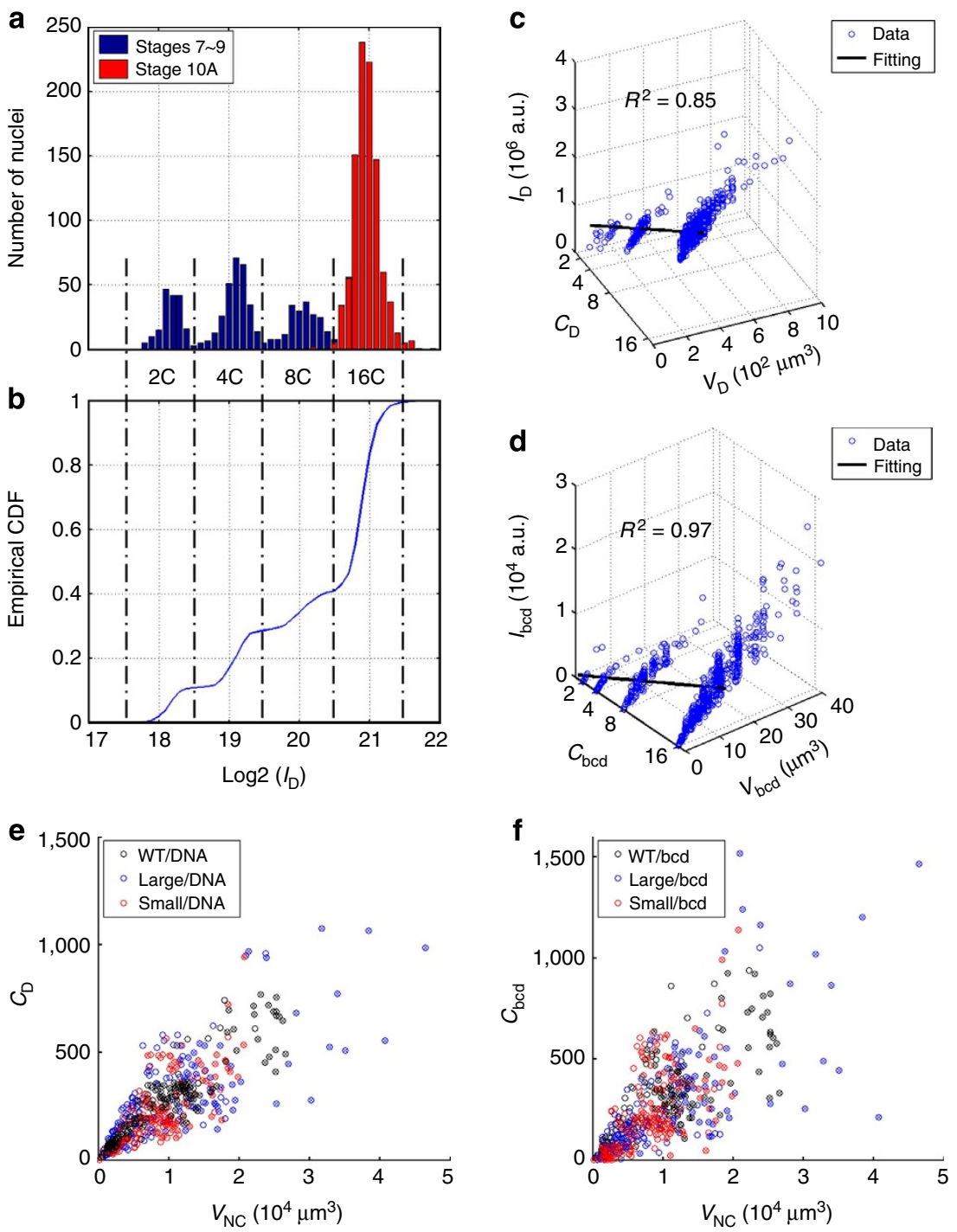

Figure 2 | Quantification of bulk nuclear DNA and bcd gene copy number in nurse cells. (a,b) Histogram (a) or empirical cumulative function (b) of nuclear DAPI intensity of the calibrating cells (1,649 follicle cells; see Methods). Dashed lines mark boundaries between subpopulations with the expected genome polyploidy of 2C, 4C, 8C and 16C. Datapoints from egg chambers at stages 7-9 or stage 10A are shown in blue and red, respectively. (c,d) Scatter plots showing a two-parameter linear fit, $I=a C+b V$, for nuclear DAPI intensity (c) or bcd DNA FISH dot intensity (d). (e,f) Scatter plots showing linear relationship between genome polyploidy equivalent (e) or bcd gene copy number (f) and nurse cell nuclear volume. Datapoints for stage $10 \mathrm{~A}$ are marked by crosses; colour code: black, blue and red for WT, large-egg line and small-egg line, respectively. The estimated polyploidy equivalents per egg chamber at stage $10 \mathrm{~A}$ are $5.7 \pm 0.5,6.1 \pm 0.5$ and $4.6 \pm 0.3 \times 10^{3}$ in WT, large-egg line and small-egg line, respectively (values are mean \pm s.d.). They are consistent with previous estimates ${ }^{44}$ considering that our calibration methods adjust for volume-dependent background intensities (see Methods). The estimated bcd gene copy numbers are given in Main text. Consistent with the analysis of egg lengths (Fig. 1b), Student's t-tests suggest significant differences in either genome polyploidy equivalent ( $P$-value $=10^{-6}$ between WT and the small-egg line, and 0.04 between WT and the large-egg line) or bcd gene copy number ( $P$ value $=0.04$ between WT and the small-egg line, and 0.12 between WT and the large-egg line). In addition, both the average polyploidy equivalents and bcd copy numbers of different lines show correlation with the average nurse cell nuclear volumes (Pearson correlation coefficients $=1.00$ and $0.99, P$ values $=0.05$ and 0.09 , respectively; $P$ values from Pearson coefficient calculation).

oogenesis ${ }^{45,49,50}$, a consensus of $n_{\mathrm{A}} \sim 3$ suggests that DNA replication for nurse cell-expressing genes is coupled with nuclear volume expansion.

Experimental evaluation of predictions of the $\mathrm{TEM}^{3} \mathrm{~S}$ model. We now consider morphogen action in instructing gene expression patterns in system $\mathbf{P}$. If $M$ at a gene's expression boundary $(\xi)$ is fixed, $\mathrm{d} M=(\partial M / \partial \xi) \mathrm{d} \xi+(\partial M / \partial L) \mathrm{d} L=0$, applying equation (2) and the exponential function of $M$ leads to

$$
\frac{d \xi}{d L / L}=\frac{n_{A}}{\Gamma \xi_{C}}\left(\xi_{C}-\xi\right),
$$

where $\mathrm{d} \xi /(\mathrm{d} L / L)$ quantifies directly how well the fluctuations in $L$ are corrected at $\xi$. We thus define $S=\mathrm{d} \xi /(\mathrm{d} L / L)$ as the scaling coefficient of an expression boundary (see legend to Fig. 5 for additional details)

Equation (4) makes three broad predictions about gene expression patterns in embryos if morphogen production (originating from maternal tissue expansion) and action could be recapitulated by the $\mathrm{TEM}^{3} \mathrm{~S}$ model: (1) there is a position, $\xi_{\mathrm{C}}$, exhibiting perfect scaling, (2) there is overscaling when $\xi<\xi_{\mathrm{C}}$ and (3) there is underscaling when $\xi>\xi_{\mathrm{C}}$. To evaluate these predictions, we performed mRNA FISH experiments to obtain the expression profiles of hunchback $(h b)$ and even-skipped (eve) 

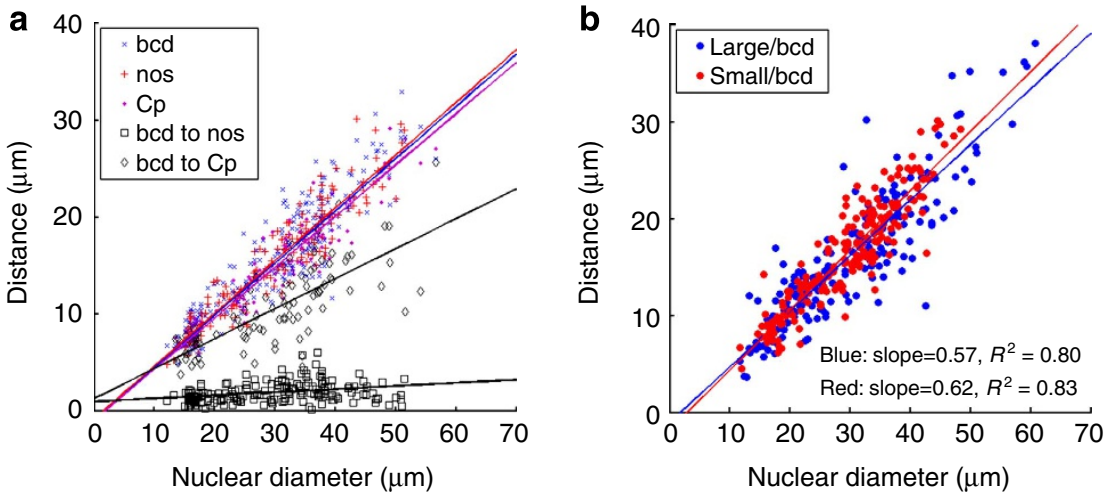

Figure 3 | Expansion of gene locus clusters in expanding nurse cell nuclei. (a) Scatter plots of the observed cluster size for individual gene loci (bcd, nos or $C p$, shown in blue, red and purple, respectively), or the observed distance between two clusters (bcd-nos or bcd-Cp, shown in black and grey, respectively), against nurse cell nuclear diameter (see Methods for definition of cluster size and distance between clusters). Data are from WT egg chambers at stages $6 \sim 10 \mathrm{~A}$. Solid lines are linear fits. Blue: $y=0.54 x-0.92 \mu \mathrm{m}, R^{2}=0.86 ;$ red: $y=0.54 x-0.84 \mu \mathrm{m}, R^{2}=0.89$; purple: $y=0.53 x-0.99 \mu \mathrm{m}, R^{2}=0.85$; black: $y=0.03 x+0.94 \mu \mathrm{m}, R^{2}=0.09$; grey: $y=0.31 x+1.25 \mu \mathrm{m}, R^{2}=0.57$. (b) The cluster size of $b c d$ DNA FISH dots against nurse cell nuclear diameter in two inbred lines. See Supplementary Fig. 6 for spatial properties of gene loci in follicle cells.
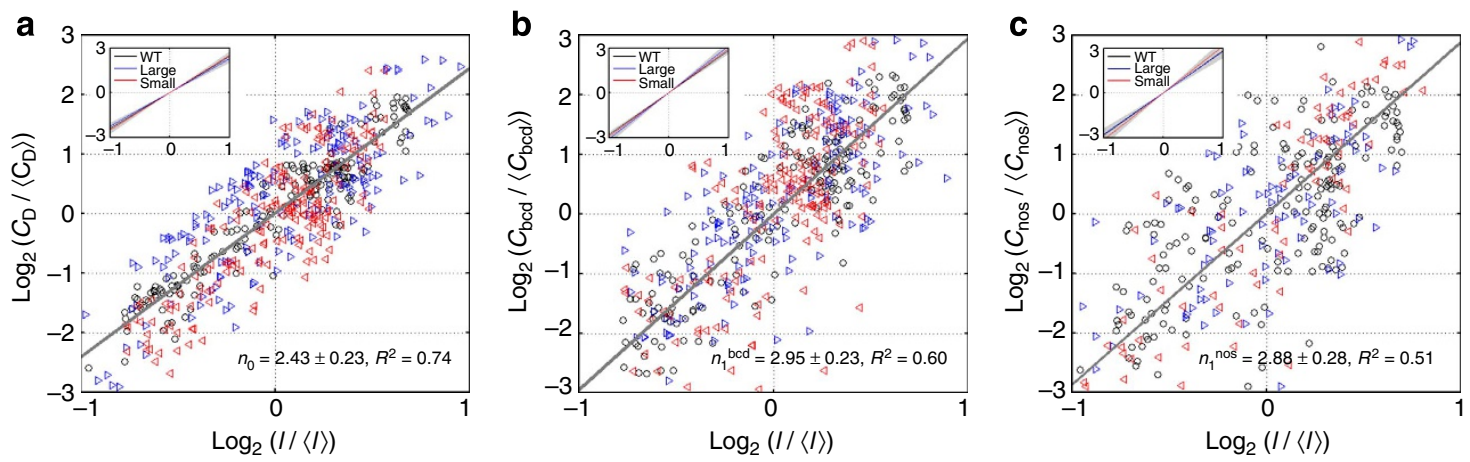

Figure 4 | Scaling power estimations for bulk nuclear DNA and gene copy numbers in nurse cells. (a-c) Log-log plots for genome polyploidy equivalent (a), bcd gene copy number (b) or nos gene copy number (c), against nurse cell nuclear diameter. Solid line is linear fit, with scaling power, $95 \% \mathrm{Cl}$ and $R^{2}$ shown. Inset shows fitting of data from individual lines. For WT (shown in black), $n_{0}=2.42 \pm 0.09, R^{2}=0.91 ; n_{1}^{\text {bcd }}=2.82 \pm 0.20, R^{2}=0.80$; $n_{1}^{\text {nos }}=2.83 \pm 0.22, R^{2}=0.76$. For the large-egg line (shown in blue): $n_{0}=2.27 \pm 0.23, R^{2}=0.67 ; n_{1}^{\text {bcd }}=3.10 \pm 0.31, R^{2}=0.67 ; n_{1}^{\text {nos }}=2.79 \pm 0.44$, $R^{2}=0.45$. For the small-egg line (shown in red): $n_{0}=2.50 \pm 0.27, R^{2}=0.74 ; n_{1}^{\text {bcd }}=2.93 \pm 0.45, R^{2}=0.60 ; n_{1}^{\text {nos }}=3.14 \pm 0.43, R^{2}=0.78$.

in embryos from the two inbred lines (Methods). As shown in Fig. $5 b$ and Supplementary Fig. $7 c$, the $h b$ boundary $(\xi=0.450)$ and the fourth boundary of eve $(\xi=0.433)$ in these embryos are closest to being perfectly scaled: $S=-0.005$ and 0.005 , respectively, whereas the more anteriorly positioned eve boundaries are progressively overscaled. These results support qualitatively the first two predictions. The third prediction is not fully supported since the predicted, progressively worsening underscaling is disrupted at $\xi \sim 0.6$ causing a trend reversal (Supplementary Fig. 7c), suggesting that patterning decisions in this part of the embryo $(\xi>0.6)$ are also sensitive to other inputs and regulatory mechanisms $24,27,51,52$

To evaluate our data quantitatively, we linearly fitted $S$ to $\xi$ for boundary positions of $h b$ and the third through sixth of eve (Fig. 5c legend). This analysis is based on an explicit assumption that these selected boundaries are specified solely by the Bcd gradient input in an idealized system $\mathbf{P}$ depicted by TEM $^{3} \mathrm{~S}$. Using experimentally measured $\Gamma=6.2$ (see Supplementary Table 2 for other measurements), we obtained $\xi_{C}=0.431$ and $n_{A}=2.64$. In essence, these two values represent the theoretically predicted properties of the embryos should they have followed explicitly the stated model, given the observed expression boundaries. Importantly, $n_{A}=2.64$ derived that this way is consistent with the values obtained from direct measurements in embryos on the actual molecular species in the linear chain (within 95\% CI of $n_{2}$ and $n_{3}$ ), further supporting TEM $^{3}$ S-derived predictions about the patterning outcomes in the specified part of the embryo.

\section{Discussion}

Our $\mathrm{TEM}^{3} \mathrm{~S}$ model provides a unified view on the properties of maternal morphogen gradients and embryonic patterning from both mechanistic and evolutionary perspectives. Extending our previous findings $14,23,25,29,53-60$ of a faithful input-output relationship between the Bcd input and the expression of its direct target gene $h b$, our current results (Fig. 5b black) show that this regulatory relationship can be recapitulated largely by the $\mathrm{TEM}^{3} \mathrm{~S}$ model. This particular relationship is likely reflective of the working of active mechanisms to allow $h b$ to respond primarily to the Bcd gradient input ${ }^{58,59}$. Thus, the performance objective of attaining a critical position by a maternal morphogen gradient may also be displayed directly by the expression boundaries of, at least in some cases, its target genes. In an idealized $\mathrm{TEM}^{3} \mathrm{~S}$ model, a patterning system benefits from having a critical position at the midpoint to allow it to receive the highest overall scaling information derived from the maternal morphogen gradient (Supplementary Note 1). There are evolutionary implications for the existence of a critical position 

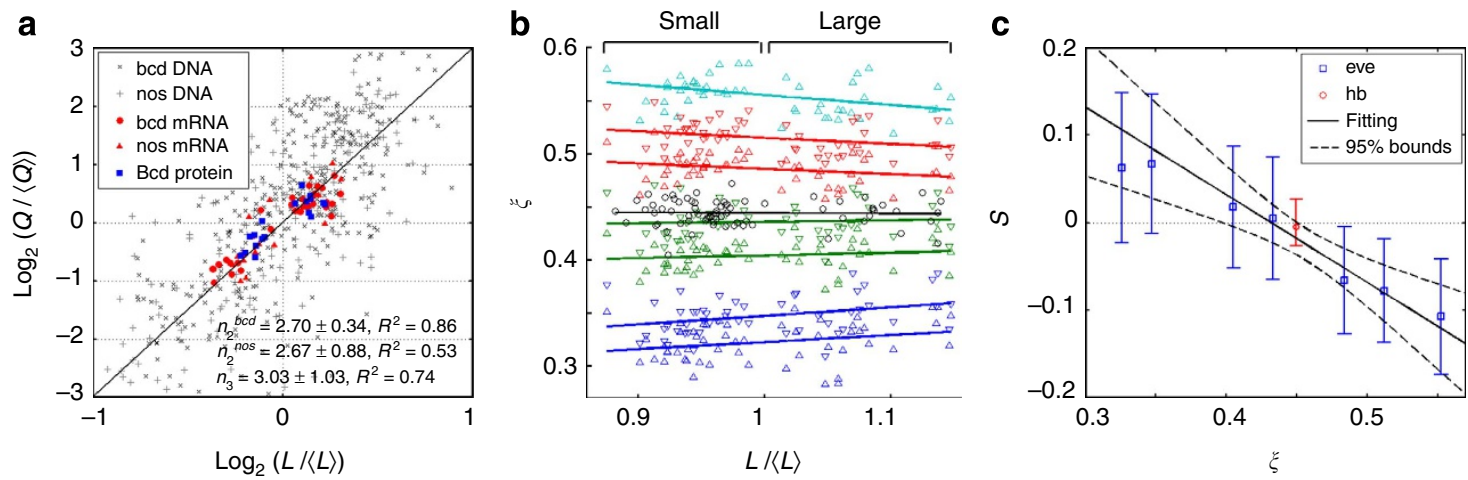

Figure 5 | Scaling power measurements in embryos and evaluations of model predictions. (a) Superimposed plots showing scaling power measurements in the two inbred lines; also shown is a reference line with a slope of 3. Datapoints for bcd DNA, nos DNA, bcd mRNA, nos mRNA and Bcd protein are shown as $\mathrm{x}$ marks, crosses, circles, diamonds and squares, respectively. (b) Scatter plot of relative positions of $h b$ (black) or the anterior 7 eve (coloured) expression boundaries in embryos from these inbred lines (see Supplementary Fig. 7 for a display showing all eve boundaries). Colour code for eve data: blue, green, red and magenta for expression stripes 1-4, respectively, with upright and downward triangles representing anterior and posterior boundaries, respectively. (c) Measured values of scaling coefficient $S$, estimated as the slope of a regression line (solid lines in $\mathbf{b}$ ), are plotted as a function of boundary position $\xi$. Error bars represent $95 \% \mathrm{Cl}$ of each fitted slope. Results for $h b$ and eve are shown in red and blue, respectively. Solid line shown is a linear fit for the selected boundaries (see text; $R^{2}=0.94$ ), and dashed lines are $95 \%$ prediction bounds of this fit. In another linear fit using boundaries within a wider range of the embryo ( $h b$ and the first through seventh of eve), we obtained consistent estimates $\left(\xi_{C}=0.422\right.$ and $n_{A}=2.13$ ) that likely have also incorporated the impact of terminal system inputs on boundaries that are closer to the anterior pole ${ }^{24,51}$. Extended discussion about scaling coefficient $S$ : Under its current definition (see text), $S=0$ denotes perfect scaling of a gene's expression boundary. If $S<0$ or $S>0$, the boundary is either under- or overscaled, respectively. This evaluation is consistent with a previous analysis ${ }^{16}$ performed under the framework of a differently defined scaling coefficient $S_{\text {Berg, }}$ as dictated by the relationship $S=\xi\left(S_{\text {Berg }}-1\right)$.

that coincides with the expression boundary of a direct Bcd target gene(s) known to have essential functions ${ }^{61-63}$. Equation (2) indicates that a meaningful critical position is achievable only if the system attribute $\Gamma$ and the scaling power of the amplitude $n_{A}$ are properly balanced with one another. Given that $n_{A}$ has a finite value dictated fundamentally by the dynamic properties of system $\mathbf{E}$ and thus is insensitive to egg size per se (see Fig. 5a and Supplementary Fig. 2), evolution of dipteran species with drastically divergent egg sizes would have to be operated under the selection pressure to preserve $\Gamma$ (Supplementary Note 1). In other words, under the $\mathrm{TEM}^{3} \mathrm{~S}$ framework, maintaining scaled and robust embryonic patterning within a species also commanded-barring a regulatory network rewiring-co-evolution of egg size and Bcd gradient properties across different species to preserve the system attribute $\Gamma$. This prediction is supported by available data ${ }^{30}$.

From the perspective of developmental biology, our $\mathrm{TEM}^{3} \mathrm{~S}$ model provides a quantitative framework to evaluate the relationship between tissue growth and tissue specification under temporal logic c. Importantly, it unifies events taking place in two distinct stages of an animal's life cycle, maternal tissue expansion and embryonic patterning. Under this framework, a robust and scaled patterning outcome of the embryo has a dynamic underpinning that is also inherent to the life cycle itself. Our results show that, meanwhile, the fundamental processes governing maternal tissue expansion impose quantifiable limits to how a developmental programme under temporal logic $\mathbf{c}$ can be constructed (Supplementary Fig. 2 and Supplementary Note 1). The other two types of temporal logics (Fig. 1a) must likely come with their own limits in connecting their respective expansion and patterning systems ${ }^{13,20,64-67}$. These limits together help to shape the forms of life on both ontogenetic and phylogenetic timescales.

Our current study also contributes to a broader on-going debate about the fundamental rules in biological scaling. Our results show that, consistent with the fact that parts of the genome are under-replicated in nurse cells ${ }^{44,49}$, the scaling power of the bulk nuclear DNA contents $n_{0}$ is smaller than that of $b c d$ gene copies $n_{1}$ (Fig. 4a) and approaches 2.25, the predicted value of the $\frac{3}{4}$-power scaling rule ${ }^{1,2}$. It remains to be known which of the scaling powers, $n_{0}$ or $n_{1}$, is the primary indicator for the fundamental mechanisms regulating endoreplication in nurse cells. A precise answer to this question will advance our knowledge of biological scaling at the level of cell cycle control in relation to nuclear size (see Supplementary Fig. 8, Supplementary Table 3 and Supplementary Note 3 for effects of expansion anisotropy on $n_{A}$ estimations). However, this layer of inquiry will not affect our current conclusion that the core quantity of the TEM ${ }^{3} S$ model, $n_{A}$, has a finite value that can be approximated-through independent measurements-by an effective isometric scaling power relationship.

Our quantification of the $b c d$ gene copy number in nurse cells reveals a peak value that resembles the peak number of nuclei in the blastoderm embryo. It is currently unknown whether this resemblance is purely coincidental. When considered in isolation, each value quantities the peak of an exponential expansion process. However, when considered together, they are systemlevel quantities that are integral to the biology of a mother and her future embryos. It remains to be investigated whether and if so, how, these particular quantities-and their relationshipmight have been influenced by evolution.

\section{Methods}

Drosophila melanogaster strains. All flies used in this study were raised on standard cornmeal-based media at $25^{\circ} \mathrm{C}$ and $60 \%$ humidity. $w^{1118}$ flies were used as the WT. Two inbred lines, no. 2.46.4 and no. 9.17.1, which have large or small eggs and are referred to as the large-egg line and small-egg line, respectively, were as described ${ }^{23}$. These lines had been derived from an artificial selection and inbreeding process on the basis of the selected traits of egg size extremes ${ }^{39}$ and were kindly provided by Cecelia Miles and Martin Kreitman. For each given set of experiments involving these two lines, all experimental and imaging procedures were performed on a side-by-side basis to permit direct comparisons.

DNA FISH in Drosophila ovaries. Our whole-mount DNA FISH procedure for the ovary was adapted from laboratory protocols of Allan Spradling ${ }^{46}$ and Terry OrrWeaver ${ }^{68}$. Briefly, newly eclosed female flies were cultured on wet yeast for 2 days and transferred to fresh wet yeast for another day. On the fourth day, ovaries were 
dissected in 400- $\mu \mathrm{l} 1 \times \mathrm{PBS}\left(137 \mathrm{mM} \mathrm{NaCl}, 2.7 \mathrm{mM} \mathrm{KCl}, 10 \mathrm{mM} \mathrm{Na}_{2} \mathrm{HPO}_{4}\right.$ and $2 \mathrm{mM} \mathrm{KH}_{2} \mathrm{PO}_{4}$ ), fixed in $4 \%$ paraformaldehyde with $0.5 \%$ Triton X-100 for $15 \mathrm{~min}$, washed with $2 \times$ SSCT $(300 \mathrm{mM} \mathrm{NaCl}, 30 \mathrm{mM}$ Sodium Citrate and $0.1 \%$ Tween20) three times and treated with $10 \mu \mathrm{g} \mathrm{ml}^{-1}$ RNaseA and RNaseT in $2 \times$ SSCT at room temperature for $30 \mathrm{~min}$. For prehybridization, the ovaries were transferred step-wise to $2 \times$ SSCT with increasing concentrations of formamide $(0,20,40$ and $50 \%$ ) and incubated in fresh $2 \times$ SSCT with $50 \%$ formamide at $37^{\circ} \mathrm{C}$ for $1 \mathrm{~h}$. The ovaries were then mixed with $40 \mu \mathrm{l}$ of probe solution consisting of $36 \mu \mathrm{l}$ $1.1 \times$ Hybridization Buffer (10\% Dextran Sulfate, $3 \times$ SSC and 50\% Formamide) and $4 \mu \mathrm{l}$ probe $(400 \mathrm{ng})$, denatured at $96^{\circ} \mathrm{C}$ for $8 \mathrm{~min}$ and chilled on ice for $5 \mathrm{~min}$, before overnight $(\sim 16 \mathrm{~h})$ incubation at $37^{\circ} \mathrm{C}$. After hybridization, ovaries were washed with $2 \times$ SSCT with $50 \%$ formamide at $37^{\circ} \mathrm{C}$ for $4 \times 15 \mathrm{~min}$, and transferred step-wise to $2 \times$ SSCT with $50,40,20$ and $0 \%$ formamide, and finally washed with $2 \times$ SSCT for $3 \times 10 \mathrm{~min}$. During the last washing step, the ovaries were brought to room temperature and further incubated with $4^{\prime}, 6$-Diamidino-2phenylindole dihydrochloride (DAPI, 1:1,000 dilution, Sigma) and Alexa Fluor 555-conjugated WGA (WGA-555 1:300 dilution, Invitrogen) at room temperature for $10 \mathrm{~min}$. After washing with $2 \times$ SSCT for $5 \times 10 \mathrm{~min}$, egg chambers were mounted in Vectashield reagent (Vector Laboratories) before imaging. Coverslip was gently pressed during mounting, when appropriate, to reduce excessive tissue thickness.

Probe preparations for DNA FISH. Probes for DNA FISH were prepared by nick translation using either the FISH Tag DNA Green Kit (Alexa Fluor 488, Life Technology) or the FISH Tag DNA Red Kit (Alexa Fluor 594, Life Technology) according to the manufacturer's instructions. Primer sequences to generate the DNA templates are listed in Supplementary Table 1. Each probe set contains six to seven individual probes $(\sim 1.3$ to $\sim 1.7 \mathrm{~kb}$ each) designed to span $>16 \mathrm{~kb}$ surrounding the target gene body but excluding the coding sequences. Unlike $b c d$ and nos, which are unique genes, there are four clusters of the chorion gene within the genome $^{68}$, DAFC-7F on chromosome X, DAFC-30B on chromosome X, DAFC$62 \mathrm{D}$ on chromosome $3 \mathrm{~L}$ and DAFC-66D on chromosome $3 \mathrm{~L}$. Our $C p$ probe set was designed to detect the 66D locus on $3 \mathrm{~L}$ including Cp18, Cp15, Cp19 and Cp16. All PCR products (from genomic DNA) used for generating probes were verified using gel electrophoresis to be of expected size as designed.

\section{Confocal imaging and documentation of intensity linearity. Images were} acquired on a Nikon A1Rsi Confocal microscope equipped with a $\times 20$ objective with $\times 3$ digital zoom. All confocal images were captured under identical settings, with the pinhole size at $1.2 \mathrm{AU}$ and the amplifier offset at 0 . A series of gain values (photomultiplier voltage (PMV)) were tested to determine the linear range of the pixel intensity for each channel (Supplementary Fig. 5). These linearity tests were on the basis of entire images to ensure that the linearity documented in Supplementary Fig. 5 is applicable to the entire intensity range including the signals from nurse cells. Images captured were 8-bit in depth and 1,024 $\times 1,024$ in the $x y$ resolution. The $z$-step size was set to match the $x y$ pixel size $(0.21 \mu \mathrm{m})$. For egg chambers that cannot be captured by a single image because their size exceeded the imaging field, $2 \times 2$ images with $15 \%$ overlap were captured and stitched.

Defining the oogenesis stage of each egg chamber. We followed established criteria to assign an egg chamber to one of the 14 stages of oogenesis ${ }^{40}$. We focused on stages $3 \sim 10 \mathrm{~A}$ for optimal size range and measurement reliability. We used the following morphological landmarks to assign an egg chamber to a developmental stage. At stage 3, the loss of oocyte nucleolus can be easily detected in the DAPI channel. At stage 4, the egg chambers are oval-shaped and DAPI staining shows a well-resolved bulbous structure in the nurse cell nuclei. The bulbous structure becomes dissolved at stage 5 and, concurrently, the homologous chromosomes begin to disassociate with multiple FISH dots becoming detectable in a nurse cell nucleus; dispersion of FISH dots continues into stage 6 as reported ${ }^{46}$. At stage 7 , the egg chamber becomes elongated, which accompanies the onset of polyploidation and enlargement of follicle cells. Egg yolk becomes first detectable at stage 8 ; this stage also marks the start of follicle cell migration, a process that continues into stage 9 . During stage 9 , border cell migration is initiated and the size of the oocyte (measured in length) is $\sim 1 / 3$ to $\sim 1 / 2$ of egg chamber length. The start of stage $10 \mathrm{~A}$ is marked by the completion of border cell migration to the boundary of oocyte and the oocyte size reaching $1 / 2$ of egg chamber length. Stage $10 \mathrm{~A}$ ends when centripetal follicle cells begin to migrate.

\footnotetext{
Measuring the egg chamber size. For each $z$-step image, we first generated a mask image combining signals from all channels above a threshold based on Otsu's method ${ }^{69}$. The length $L$ was defined as the longest distance between two pixels of each egg chamber (that is, the major axis or pseudo AP axis) on the maximum projection of all $z$-sections. The height $H$ was then defined as the longest distance perpendicular to $L$ on the same projection. $L$ and $H$ were measured automatically using MATLAB (Math Works); for egg chambers that are in physical contact with each other, manual adjustments were made. We performed five repeated measurements to obtain an average and found that a typical measurement error was $\sim 1 \%$.
}

Estimating the detection efficiency of DNA FISH. Follicle cells were used to estimate the detection efficiency because of their large numbers and relatively uniform DNA FISH intensities. Among the 2,695 identifiable follicle cell nuclei from $30 w^{1118}$ egg chambers at stages $7 \sim 10 \mathrm{~A}, 2,373$ of them had a single $b c d$ fluorescent dot detected, 297 of them had two dots and 25 of them had none. This suggests a detection efficiency of $\sim 99 \%$ under our experimental procedure, with a high frequency $(\sim 89 \%)$ of association between homologous chromosomes in follicle cell nuclei. Similar results were obtained for two other loci analysed in this study, nos and $C p$. Before chromosome dispersion, each nurse cell nucleus had on average $0.91 \pm 0.27,0.94 \pm 0.33$ and $0.89 \pm 0.21$ identifiable FISH dots for the loci of $b c d$, nos and $C p$, respectively, on the basis of 82 nurse cells analysed (from $10 w^{1118}$ egg chambers at stages $2 \sim 5$ ). After dispersion, up to 32 FISH dots were detectable per nurse cell nucleus, with an average of $21.0 \pm 6.3,21.3 \pm 6.9$ and $21.1 \pm 8.0$ for $b c d, n o s$ and $C p$, respectively, on the basis of 335 nurse cells from 39 egg chambers analysed. This suggests an incomplete dissociation of polytene chromosomes in our whole-mount tissues that were processed under conditions to maximally preserve their native spatial features (see also Main text).

Measuring the statistics of DAPI and FISH signals. Thresholds were determined, on the basis of the following two considerations, to identify nuclei and FISH dots: (1) the intensity threshold was sufficiently high to eliminate false objects arisen from stochastic noise; (2) the threshold was high enough to avoid fusion of neighbouring objects. These two tests led to a stable profile of the number of identified objects against the chosen threshold within a range. Thresholds were then chosen automatically within the stable region where the first-order slope is equal to 0 . The sizes and the intensities of nuclei and FISH dots were quantified by two methods. In the first method, the volume of a nucleus or a FISH dot was defined as the number of voxels within each identified object, and the intensity was defined as the aggregated intensity of all voxels within each identified object. Background values of either DAPI or FISH signals were acquired from the mean voxel intensities in regions away from nuclei. In the other method, the centre voxel of each identified object was enclosed by a smallest sphere with a volume $(V)$, and the total raw intensity $(I)$ was aggregated from all voxels within the sphere. We then fitted data to a two-parameter linear model, $I=a C+b V$, where $C$ is the polyploidy number (for DAPI) or gene copy number (for FISH); see below for details about data calibration. The choice of shape and size of the enclosing sphere did not alter our conclusions and the linearity between the sizes or the intensities measured by two independent methods further confirmed the reliability of our quantification methods.

Measuring the spatial distributions within nuclei. The distance between a FISH dot and the nuclear envelope of a follicle cell was measured as the shortest Euclidean distance from any voxel within the FISH dot to any voxel on the boundary of the identified nucleus (Supplementary Fig. 6). The distance between two FISH dots within a follicle cell nucleus was measured as the Euclidean distance between the centre voxels of the two FISH dots (Supplementary Fig. 6). The one-dimensional (1D) size of a cluster of FISH dots of a gene locus was measured as the longest distance between any two dots within the cluster (Fig. 3a,b). The distance between the clusters of two gene loci was measured as the Euclidean distance between the weighted centre positions of the two clusters. Two pairs of gene loci (all on chromosome 3) were evaluated for their spatial relationships, and their intervening DNA lengths are: $b c d$-nos (Fig. 3a, black squares), $12.4 \mathrm{Mbps}$ (both on 3R); $b c d$-Cp (Fig. 3a, grey diamonds) and 19.3 Mbps (on two different arms of chromosome 3).

Calibrating the copy numbers of DNA contents and gene locus. We used data from the identified follicle cells in stages $7 \sim 10$-A egg chambers for calibration purposes. The distribution of DAPI intensity of these follicle cells was plot on a $\log 2$ scale, which uncovers four well-separated subpopulations. We assigned, as before ${ }^{55}$ the expected $2 \mathrm{C}, 4 \mathrm{C}, 8 \mathrm{C}$ and $16 \mathrm{C}$, respectively, to each of the four largest subpopulations in the distribution of raw DAPI intensity of follicle cells (Fig. 2a). The approximately twofold-DAPI intensity difference between any given two adjacent subpopulations validates this assignment ${ }^{45}$. The boundary intensities between two adjacent subpopulations were specified by the values in the valleys and acquired as the intensity index where the first-order differentiation of the probability density function is 0 . In our calibration, we grouped the follicle cells according to their DAPI intensities $\left(I_{\mathrm{D}}\right)$ and their assigned DNA contents expressed as a polyploidy number $\left(C_{\mathrm{D}}\right)$. Then we fitted the data to a two-parameter linear model, $I_{\mathrm{D}}=a_{1} C_{\mathrm{D}}+b_{1} V_{\mathrm{D}}$. For DNA FISH data, we assumed that in follicle cells, the copy number of the gene locus $\left(C_{\mathrm{g}}\right)$ is the same as $C_{\mathrm{D}}$ and performed the fitting with $I_{\mathrm{g}}=a_{2} C_{\mathrm{g}}+b_{2} V_{\mathrm{g}}$. With the parameters $a$ and $b$ obtained for each set of experiments, we converted the detected intensity data to estimates of both the bulk DNA contents expressed as the polyploidy equivalent and the copy number of a gene locus in a nurse cell. See Supplementary Fig. 5 and the confocal imaging section above for discussions about intensity range and linearity documented experimentally.

RNA FISH in Drosophila embryos. RNA FISH in embryos was performed using fluorescence-labelled probes or digoxigenin-labelled probes detected by an antidig antibody and a fluorescence-labelled secondary antibody ${ }^{58}$. In our current study, probes were prepared from cDNA plasmids or genomic PCR products; for direct 
fluorescence labelling, the FISH Tag RNA kit (Alexa Fluor 488, Life Technology) was used. Embryos used in $b c d$ and nos mRNA FISH were from 0- to 1-h collections and those used in $h b$ and eve mRNA FISH from 0- to 3- and 0- to 4-h collections, respectively. Imaging was performed under the Zeiss Imager Z1 ApoTome microscope with a Zeiss Plan $\times 10$ Aprochromat objective. Imaging acquisition was performed under linear settings and data analysis (MATLAB, Math Works) was on the basis of fluorescent intensities extracted from the cytoplasmic layer of midsagittal images as a function of AP position (for $h b$ and eve) s. $^{58}$ or as epifluorescence intensities (for $b c d$ and nos) ${ }^{23}$.

TEM ${ }^{3} \mathbf{S}$ model and other theoretical considerations. Supplementary Notes 1-4 provide a formal presentation of the $\mathrm{TEM}^{3} \mathrm{~S}$ model and other related aspects of theoretical considerations.

\section{References}

1. Kleiber, M. Body size and metabolism. Hilgardia 6, 315-353 (1932).

2. West, G. B., Brown, J. H. \& Enquist, B. J. The fourth dimension of life: fractal geometry and allometric scaling of organisms. Science 284, 1677-1679 (1999).

3. Reich, P. B., Tjoelker, M. G., Machado, J. L. \& Oleksyn, J. Universal scaling of respiratory metabolism, size and nitrogen in plants. Nature 439, 457-461 (2006).

4. Enquist, B. J. et al. Biological scaling: does the exception prove the rule? Nature 445, E9-E10 (2007).

5. Kolokotrones, T., Van, S., Deeds, E. J. \& Fontana, W. Curvature in metabolic scaling. Nature 464, 753-756 (2010).

6. MacKay, N. J. Mass scale and curvature in metabolic scaling. Comment on: T. Kolokotrones et al., curvature in metabolic scaling, Nature 464 (2010) 753-756. J. Theor. Biol. 280, 194-196 (2011).

7. Deeds, E. J., Savage, V. \& Fontana, W. Curvature in metabolic scaling: a reply to MacKay. J. Theor. Biol. 280, 197-198 (2011).

8. Waddington, C. H. Canalization of development and the inheritance of aquired characters. Nature 150, 563-565 (1942).

9. Patel, N. H. \& Lall, S. Precision patterning. Nature 415, 748-749 (2002).

10. Lander, A. D. Pattern, growth, and control. Cell 144, 955-969 (2011).

11. Houchmandzadeh, B., Wieschaus, E. \& Leibler, S. Establishment of developmental precision and proportions in the early Drosophila embryo. Nature 415, 798-802 (2002)

12. Lott, S. E., Kreitman, M., Palsson, A., Alekseeva, E. \& Ludwig, M. Z. Canalization of segmentation and its evolution in Drosophila. Proc. Natl Acad. Sci. USA 104, 10926-10931 (2007).

13. Crickmore, M. A. \& Mann, R. S. The control of size in animals: insights from selector genes. BioEssays 30, 843-853 (2008).

14. He, F. et al. Probing intrinsic properties of a robust morphogen gradient in Drosophila. Dev. Cell 15, 558-567 (2008).

15. Manu et al. Canalization of gene expression in the Drosophila blastoderm by gap gene cross regulation. PLoS Biol. 7, e1000049 (2009).

16. de Lachapelle, A. M. \& Bergmann, S. Precision and scaling in morphogen gradient read-out. Mol. Syst. Biol. 6, 351 (2010).

17. Ben-Zvi, D., Shilo, B. Z. \& Barkai, N. Scaling of morphogen gradients. Curr. Opin. Genet. Dev. 21, 704-710 (2011).

18. Wartlick, O., Mumcu, P., Julicher, F. \& Gonzalez-Gaitan, M. Understanding morphogenetic growth control -- lessons from flies. Nature reviews. Mol. Cell Biol. 12, 594-604 (2011).

19. Umulis, D. M. \& Othmer, H. G. Mechanisms of scaling in pattern formation. Development 140, 4830-4843 (2013)

20. Yang, X. \& Xu, T. Molecular mechanism of size control in development and human diseases. Cell. Res. 21, 715-729 (2011).

21. Wartlick, O. et al. Dynamics of Dpp signaling and proliferation control. Science 331, 1154-1159 (2011).

22. Schwank, G., Yang, S. F., Restrepo, S. \& Basler, K. Comment on 'Dynamics of dpp signaling and proliferation control'. Science 335, 401 (2012).

23. Cheung, D., Miles, C., Kreitman, M. \& Ma, J. Scaling of the Bicoid morphogen gradient by a volume-dependent production rate. Development 138, 2741-2749 (2011).

24. Porcher, A. \& Dostatni, N. The bicoid morphogen system. Curr. Biol. 20, R249-R254 (2010).

25. Liu, J., He, F. \& Ma, J. Morphogen gradient formation and action: insights from studying Bicoid protein degradation. Fly. (Austin. Tex). 5, 242-246 (2011).

26. Dalessi, S., Neves, A. \& Bergmann, S. Modeling morphogen gradient formation from arbitrary realistically shaped sources. J. Theor. Biol. 294, 130-138 (2012).

27. Jaeger, J., Manu \& Reinitz, J. Drosophila blastoderm patterning. Curr. Opin. Genet. Dev. 22, 533-541 (2012).

28. Grimm, O., Coppey, M. \& Wieschaus, E. Modelling the Bicoid gradient. Development 137, 2253-2264 (2010).

29. Cheung, D., Miles, C., Kreitman, M. \& Ma, J. Adaptation of the length scale and amplitude of the Bicoid gradient profile to achieve robust patterning in abnormally large Drosophila melanogaster embryos. Development 141, 124-135 (2014).
30. Gregor, T., Bialek, W., de Ruyter van Steveninck, R. R., Tank, D. W. \& Wieschaus, E. F. Diffusion and scaling during early embryonic pattern formation. Proc. Natl Acad. Sci. USA 102, 18403-18407 (2005).

31. Bergmann, S. et al. Pre-steady-state decoding of the Bicoid morphogen gradient. PLoS Biol. 5, e46 (2007).

32. Coppey, M., Berezhkovskii, A. M., Kim, Y., Boettiger, A. N. \& Shvartsman, S. Y. Modeling the bicoid gradient: diffusion and reversible nuclear trapping of a stable protein. Dev. Biol. 312, 623-630 (2007).

33. Gregor, T., Tank, D. W., Wieschaus, E. F. \& Bialek, W. Probing the limits to positional information. Cell 130, 153-164 (2007).

34. Kerszberg, M. \& Wolpert, L. Specifying positional information in the embryo: looking beyond morphogens. Cell 130, 205-209 (2007).

35. Tostevin, F., ten Wolde, P. R. \& Howard, M. Fundamental limits to position determination by concentration gradients. PLoS Comput. Biol. 3, e78 (2007).

36. Jaeger, J. \& Martinez-Arias, A. Getting the measure of positional information. PLoS Biol. 7, e81 (2009).

37. Okabe-Oho, Y., Murakami, H., Oho, S. \& Sasai, M. Stable, precise, and reproducible patterning of bicoid and hunchback molecules in the early Drosophila embryo. PLoS Comput. Biol. 5, e1000486 (2009).

38. Deng, J., Wang, W., Lu, L. J. \& Ma, J. A two-dimensional simulation model of the bicoid gradient in Drosophila. PLoS ONE 5, e10275 (2010).

39. Miles, C. M. et al. Artificial selection on egg size perturbs early pattern formation in Drosophila melanogaster. Evolution 65, 33-42 (2011).

40. Spradling, A. C. in The Development of Drosophila melanogaster (eds Bates, M. \& Martinez-Arias, A.) 1-70 (Cold Spring Harbor Press, 1993).

41. Naora, H. \& Montell, D. J. Ovarian cancer metastasis: integrating insights from disparate model organisms. Nat. Rev. Cancer 5, 355-366 (2005).

42. Bastock, R. \& St Johnston, D. Drosophila oogenesis. Curr. Biol. 18, R1082-R1087 (2008).

43. Klusza, S. \& Deng, W. M. At the crossroads of differentiation and proliferation: precise control of cell-cycle changes by multiple signaling pathways in Drosophila follicle cells. BioEssays 33, 124-134 (2011).

44. Hammond, M. P. \& Laird, C. D. Chromosome structure and DNA replication in nurse and follicle cells of Drosophila melanogaster. Chromosoma 91, 267-278 (1985).

45. Calvi, B. R., Lilly, M. A. \& Spradling, A. C. Cell cycle control of chorion gene amplification. Genes Dev. 12, 734-744 (1998).

46. Dej, K. J. \& Spradling, A. C. The endocycle controls nurse cell polytene chromosome structure during Drosophila oogenesis. Development 126, 293-303 (1999).

47. Marshall, W. F. et al. Interphase chromosomes undergo constrained diffusional motion in living cells. Curr. Biol. 7, 930-939 (1997).

48. Petkova, M. D., Little, S. C., Liu, F. \& Gregor, T. Maternal origins of developmental reproducibility. Curr. Biol. 24, 1283-1288 (2014).

49. Edgar, B. A. \& Orr-Weaver, T. L. Endoreplication cell cycles: more for less. Cell 105, 297-306 (2001).

50. de Nooij, J. C., Graber, K. H. \& Hariharan, I. K. Expression of the cyclindependent kinase inhibitor Dacapo is regulated by cyclin E. Mech. Dev. 97, 73-83 (2000).

51. Lohr, U., Chung, H. R., Beller, M. \& Jackle, H. Bicoid--morphogen function revisited. Fly (Austin. Tex). 4, 236-240 (2010).

52. Chen, H., Xu, Z., Mei, C., Yu, D. \& Small, S. A system of repressor gradients spatially organizes the boundaries of Bicoid-dependent target genes. Cell 149, 618-629 (2012).

53. He, F. et al. Shaping a morphogen gradient for positional precision. Biophys. J. 99, 697-707 (2010).

54. He, F. et al. Distance measurements via the morphogen gradient of Bicoid in Drosophila embryos. BMC Dev. Biol. 10, 80 (2010).

55. He, F., Ren, J., Wang, W. \& Ma, J. A multiscale investigation of bicoiddependent transcriptional events in Drosophila embryos. PLoS ONE 6, e19122 (2011).

56. He, F., Ren, J., Wang, W. \& Ma, J. Evaluating the Drosophila Bicoid morphogen gradient system through dissecting the noise in transcriptional bursts. Bioinformatics 28, 970-975 (2012).

57. He, F. \& Ma, J. A spatial point pattern analysis in Drosophila blastoderm embryos evaluating the potential inheritance of transcriptional states. PLoS ONE 8, e60876 (2013).

58. Liu, J. \& Ma, J. Dampened regulates the activating potency of Bicoid and the embryonic patterning outcome in Drosophila. Nat. Commun. 4, 2968 (2013).

59. Liu, J. \& Ma, J. Uncovering a dynamic feature of the transcriptional regulatory network for anterior-posterior patterning in the Drosophila embryo. PLoS ONE 8, e62641 (2013).

60. Liu, J. \& Ma, J. Fates-shifted is an F-box protein that targets Bicoid for degradation and regulates developmental fate determination in Drosophila embryos. Nat. Cell Biol. 13, 22-29 (2011).

61. Wimmer, E. A., Carleton, A., Harjes, P., Turner, T. \& Desplan, C. Bicoidindependent formation of thoracic segments in Drosophila. Science 287, 24762479 (2000). 
62. Hulskamp, M., Pfeifle, C. \& Tautz, D. A morphogenetic gradient of hunchback protein organizes the expression of the gap genes Kruppel and knirps in the early Drosophila embryo. Nature 346, 577-580 (1990).

63. Yu, D. \& Small, S. Precise registration of gene expression boundaries by a repressive morphogen in Drosophila. Curr. Biol. 18, 868-876 (2008).

64. Averbukh, I., Ben-Zvi, D., Mishra, S. \& Barkai, N. Scaling morphogen gradients during tissue growth by a cell division rule. Development 141, 2150-2156 (2014).

65. Wartlick, O., Julicher, F. \& Gonzalez-Gaitan, M. Growth control by a moving morphogen gradient during Drosophila eye development. Development 141, 1884-1893 (2014)

66. Muller, P., Rogers, K. W., Yu, S. R., Brand, M. \& Schier, A. F. Morphogen transport. Development 140, 1621-1638 (2013).

67. Kicheva, A. et al. Coordination of progenitor specification and growth in mouse and chick spinal cord. Science 345, 1254927 (2014).

68. Claycomb, J. M., Benasutti, M., Bosco, G., Fenger, D. D. \& Orr-Weaver, T. L. Gene amplification as a developmental strategy: isolation of two developmental amplicons in Drosophila. Dev. Cell 6, 145-155 (2004).

69. Otsu, N. A threshold selection method from gray-level histograms. IEEE. Trans. Syst. Man. Cybern. 9, 62-66 (1979).

\section{Acknowledgements}

We thank Cecelia Miles and Martin Kreitman for providing the inbred lines used in this study, Dianne Williams in Allan Spradling's laboratory and Brian Hua in Terry

Orr-Weaver's laboratory for sharing their DNA FISH protocols, Satoshi Namekawa,
Matt Kofron and Junbo Liu for discussions and technical assistance, and Tim Saunders for critical reading of the manuscript. This work was supported in part by 1R01GM101373 from NIH and IOS-0843424 from NSF (to J.M.). R.J. is supported by grants from the NSFC (31271573) and the 973 programme (2012CB825504).

\section{Author contributions}

F.H., C.W. and J.M. developed the concepts and experimental approaches. C.W. developed the whole-mount DNA FISH and confocal imaging procedures. C.W. and H.W. performed experiments and generated data. F.H. developed quantitative methods, performed data analysis and generated all figures. F.H. and J.M. initiated and developed the concepts for the $\mathrm{TEM}^{3} \mathrm{~S}$ model. F.H. had a leading role in the formulation and writing of the $\mathrm{TEM}^{3} \mathrm{~S}$ model and all the theoretical parts of Supplementary Information. D.C. and R.J. contributed resources. F.H. and J.M. wrote the paper and all approved the paper.

\section{Additional information}

Supplementary Information accompanies this paper at http://www.nature.com/ naturecommunications

Competing financial interests: The authors declare no competing financial interests.

Reprints and permission information is available online at http://npg.nature.com/ reprintsandpermissions/

How to cite this article: He, F. et al. Fundamental origins and limits for scaling a maternal morphogen gradient. Nat. Commun. 6:6679 doi: 10.1038/ncomms7679 (2015). 